\title{
Tapology: A Game-Based Platform to Facilitate E-Health and E-Inclusion
}

\author{
Kenneth C. Scott-Brown ${ }^{1}$, Julie Harris ${ }^{2}$, Anita Simmers ${ }^{3}$, Mhairi Thurston ${ }^{1}$, \\ Malath Abbas ${ }^{4}$, Tom de Majo ${ }^{4}$, Ian Reynolds ${ }^{4}$, Gareth Robinson ${ }^{4}$, Iain Mitchell ${ }^{1}$, \\ Dan Gilmour ${ }^{5}$, Santiago Martinez ${ }^{6}$, and John Isaacs ${ }^{5, *}$ \\ ${ }^{1}$ School of Social and Health Sciences, Abertay University, Dundee, UK \\ ${ }^{2}$ School of Psychology and Neuroscience, University of St Andrews, Fife, UK \\ ${ }^{3}$ Vision Sciences Department, Glasgow Caledonian University, Glasgow, UK \\ ${ }^{4}$ Quartic Llama, Bannerman House, 27 South Tay Street, Dundee, UK \\ ${ }^{5}$ School of Science, Engineering \& Technology, \\ Abertay University, Dundee, UK \\ ${ }^{6}$ Center for eHealth and Healthcare Technology, \\ University of Agder, Grimstad, Norway \\ \{k.scott-brown, M. Thurston, d.gilmour, j.isaacs\}@abertay.ac.uk, \\ jh81est-andrews.ac.uk, \\ Anita.Simmers@gcu.ac.uk, \\ \{mal, gaz, ian, tom\} @quarticllama.com, \\ izmitchell@dundee.ac.uk, \\ santiago.martinez@uia.no
}

\begin{abstract}
We have developed a tablet computer game app for low vision users that can be used to introduce a platform for gaming, internet and visual rehabilitation to older users who have not had prior experience with information communication technology (ICT). Our target user group is people diagnosed with Age Related Macular Degeneration (AMD). The primary goal of the app is to present a fun and engaging means for participants to engage with Information Communication Technology (ICT). A long-term goal of the project is to build a platform to gather data on current and on-going visual function by creating a suite of games that could generate sufficient regular visual engagement to enable perceptual learning in the preserved peripheral retina that is spared in AMD. The inclusive design process took into consideration the perceptual and cognitive constraints of the user group in. The 'Tapology@' app was formally launched at a large computer games festival where we gathered data from a range of users to inform the development of the gameplay. The initial results and feedback inform the ultimate goal of creating a suite of applications that have a wide social and geographic reach to promote and inform e-inclusion and e-health.
\end{abstract}

Keywords: E-Health; E-Inclusion, Games, co-design, accessible design, Mobile HCI.

* Corresponding author.

C. Stephanidis and M. Antona (Eds.): UAHCI/HCII 2014, Part III, LNCS 8515, pp. 368-377, 2014.

(c) Springer International Publishing Switzerland 2014 


\section{Introduction}

Few of us can imagine the impact and sense of loss associated with a diagnosis of an irreversible sight-loss condition such as Age-Related Macular Degeneration (AMD). Recently, one of the authors [1] has documented the social and emotional impact of making the transition from sightedness to blindness using to a series of interviews with clients who have undergone such a journey. It transpires that there is often a temporal disconnect between diagnosis, the behavioural 'point of impact' of sight loss, and the point of clinical intervention. In this gap, there is an opportunity to develop interventions designed to address the dramatic changes imminent in life prior to the 'point of impact' of blindness (where dramatic changes in lifestyle become unavoidable). Although treatments are developing, as yet conditions such as AMD have no 'cure', this should not prevent clinicians and the third sector from being able to offer a constructive set of interventions. A wide variety of assistive technology is available, yet Thurston [1] has identified that there is often reluctance to take on board such technology early in the disease trajectory since this would be an outward sign of defeat.

In view of the reluctance of newly diagnosed clients to move to overt and potentially stigmatising assistive technologies, the current project is aimed at providing an inclusive and engaging intervention that can promote e-inclusion and adoption of assistive technologies. This need is amplified by the well-documented lack of uptake of Information Communication Technology (ICT) in older groups [2]. The resulting intervention is based on an interactive tablet computer 'app' designed to challenge different cognitive and visual components of the users visual system in a fun and engaging way that can facilitate interaction with ICT and indirectly engender a broader awareness of the assistive technologies embedded in tablet computers. There is a longer term goal to use this intervention to develop a specific vision training protocol to test the hypothesis that peripheral vision in AMD patients might be subject to visual training to improve visual function [3]. This would first require a palatable nonclinical vehicle for regular interaction.

The visual experience of AMD is one of loss of central visual acuity. Initially this is not subject to conscious awareness, but it progresses, meaning that items must be scaled up in size and effectively presented in periphery in order to be visible. Our peripheral vision is not as good as central vision, but tantalising results from video games research suggest that it might be possible to 'train up' peripheral vision to improve visual function [4,5]. These experiments indicate that in experienced video games players, the so-called Useful Field of View is increased compared to non-video gamers. These results are typically found with players of military-based first-person 'shooter' games. However, such combat based titles do not fit the demographic of the target patient group which is the over 60s. However, one of us has shown that in the case of Amblyopia, much simpler games such as Tetris can have beneficial effects on visual performance [6]

The crucial skill in developing the AMD patients' transition to reading with low vision is their ability to recruit peripheral vision using their so-called Preferred Retinal Locus (PRL), the region of spared vision [7]. This skill requires the ability to fixate 
steadily in one location, yet attend to a peripheral one. It is precisely these sorts of skills that characterize modern video game play, and this indicates the potential of developing a games based approach to fixation training. Previously it has been argued that the recognition of users personal goals is crucial for accessible development for occasional users or users with no prior experience of ICT [8]. For example, the primary goals for the users in the ICT contexts discussed may not be primarily of a clinical nature. Rather, the ability to communicate with friends and family or to share activities would likely be a primary goal. With this in mind, an assistive app will still benefit from its ability to address primary goals of the user rather than secondary goals. This is the justification for the game-based approach: to make the experience enjoyable.

\section{Development}

As pointed out by Keates [9] the profile of users must be considered during the design of accessible apps. Thus, in collaboration with the clients from Fife Society for the Blind, initial research and development led to a design project with a number of patients at various stages of AMD. During the meeting, the co-design methodology [10] was used to incorporate user feedback to help shape the game. By meeting and listening to the target audience it helped the design team to understand their particular needs, gain insight into AMD and helped us to gather information based on habits and daily life, which ultimately helped us design an appropriate game. During the design meeting basic paper prototypes were built to help visualise the design and user feedback was recorded to help steer the process. Additional immersive techniques, such as sight simulation spectacles were used by the team to assist in the development of the prototypes in line with previous recommendations [11] for inclusive design.

The initial step was to work out what form the app would take, with the original plan being some sort of sight based game. Approximately a week was spent making a number of very quick, simple, prototypes; this would serve as a platform to test internally and provide a clearer path forward. The prototypes were analysed based on visual aesthetic, usability, and data collection potential to help further analysis on user behaviour and playing patterns. Around nine simple prototypes were made, but due to the potential complexity of AMD none of these prototypes on their own were thought to cover the range of impairments. Instead, the five prototypes with the most potential were collated into a mini-game collection. Together they would better cover the different aspects and issues of e-inclusion and engagement.

The suite of mini-games suite was developed using the Unity game engine (C) Unity Technologies). Team roles ranged from project management and game design to development and testing. This decision to use the Unity development environment came down to past experience of working with the game engine developing rapid prototypes and its porting ability making it easy to deploy to a range of different platforms. The suite was named 'Tapology' (C Abertay University) (Fig. 1) and was designed for the iPad ( $($ ) Apple Corporation) due to its large screen size and touch interface. By focusing on a single device it was possible to develop a robust prototype. By using the Unity game engine the app can easily be deployed on other tablet or touch screen devices currently available on the market. 

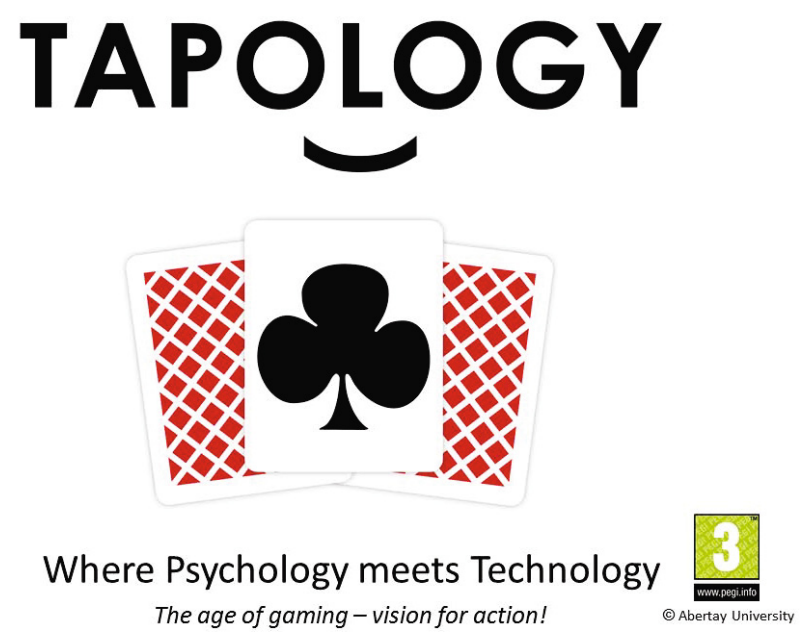

Fig. 1. The 'Tapology' branding allows for the addition of additional minigames, all based around the concept of tap based gesture interaction

Five mini-games were developed: Reaction, Target Tap, Colour Change, Card Match, and Pouring. Each of the mini-games is backed by a unique colour, to differentiate it from the others. Throughout each game, bright colours and strong contrasts are used to make all the presented information as clear as possible for any user.

The 'Pouring' mini-game illustration (Fig 2) shows how the game developers brought to life the concept from one of the co-design groups. In essence, the game involves collecting rain from the increasingly rapidly moving clouds to develop the garden. By focusing on a relevant, skill, the training aspects of visually guided action can be developed in a manner relevant and engaging for the user group. It is worth noting that the game 'mechanic' is in essence a reverse 'space invaders' game. In discussion, there was a strong sense that mainstream video games did not identify the appropriate demographic touchstones, nor did they address required skills for lowvision users. Nevertheless, older users regularly play games such as Scrabble, Cards, Mah Jong etc., despite not seeing themselves as 'gamers' in the modern sense.

The mini-games are kept short, and most increase in difficulty over the course of the game. Once the user finishes a game, they are awarded a score out of three stars, similar to a number of popular mobile games depending on how well they performed. This score, along with other relevant statistics are sent to a cloud-based data account so that they can be later reviewed. Data to be tracked includes the player's score, their reaction times and the current difficulty settings. Importantly for the player, this helped to add replay value to the experience and a sense of competition, which helped to make the experience social - a priority that was identified in the co-design meetings.

A key goal of the app is visual assessment of clients, and this is provided by a 'Target Tap' mini-game. In this game, the user must as quickly as possible touch the centre of a circular target. This is the clearest link to visual assessment that forms the backbone of the long-term goal of patient ownership concept of the app. By 
personalising visual assessment and taking it out of the clinical setting, it becomes possible to reduce the stressful component of testing. Further, it opens up the possibility for a longitudinal component of testing, not otherwise practical with fixed clinical testing locations due to the cost of travel and clinician time. In the context of the North Sea Region of Europe, this mobile aspect of testing is particularly useful since distances to travel to clinics in Scotland, Norway and Sweden become prohibitively obstructive.

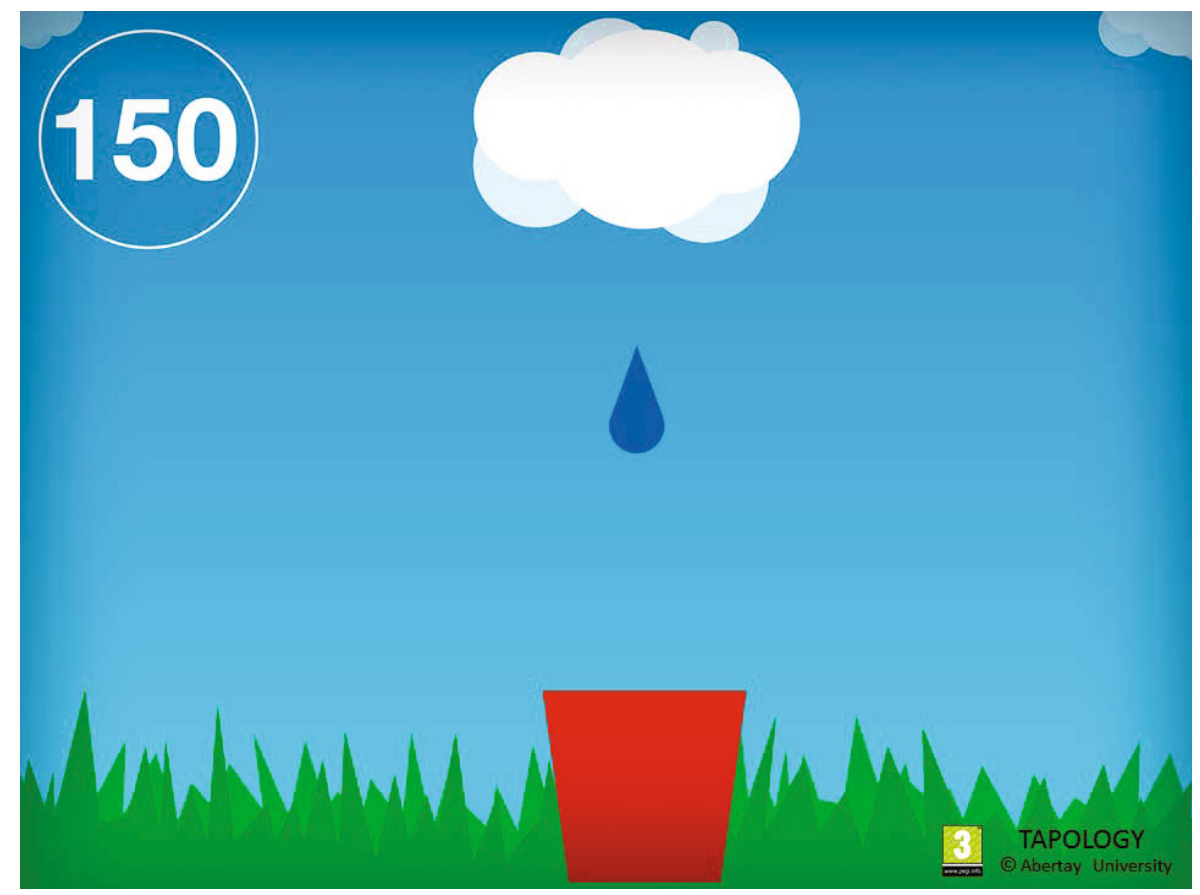

Fig. 2. The 'Pouring' game requires the user to use their finger to drag the bucket left and right and catch the rain drops. The bucket moves more quickly at each level to increase playability.

In the mini-game 'Card Match' (Fig. 3) where participants must fixate the central card during an exposure of an array of cards, and then subsequently locate and tap the correct peripheral card. This game involves steady gaze fixation and good short-term memory.

'Reaction' is a game requiring the user to respond with the appropriate interface gesture, the targets appear randomly on the screen requiring distributed attention. The aim is to reinforce the tactile skills required to operate a capacitive touch screen - an effective 'tap'.

'Colour Change', requires the user to focus on the centre of the screen to identify a central objective colour, and then 'tag' similarly coloured elements as they approach the centre. Loosely based on the concept of radar, the objective is sustained vigilance with visually guided action. 


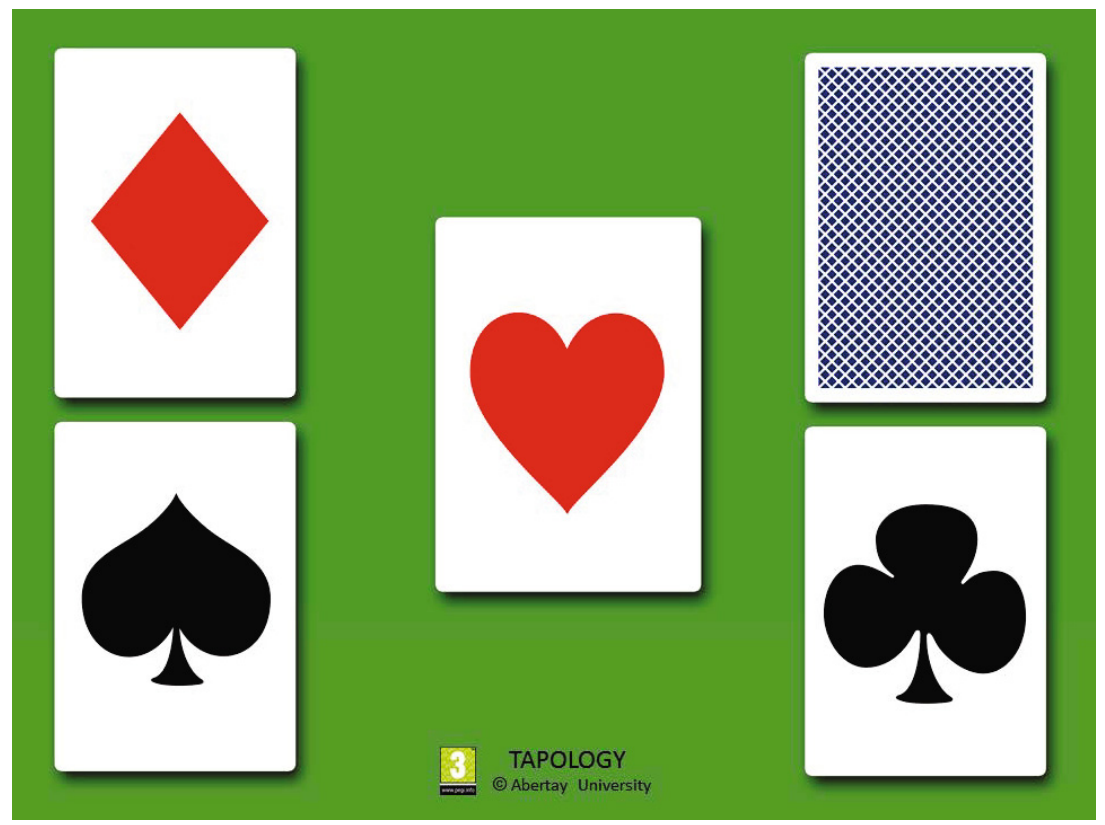

Fig. 3. Development still from 'Card Match' Memory and stable fixation are required for the Card Match game

\section{$3 \quad$ First User Trial}

\subsection{Participants}

User trials involved 60 participants, 37 male, 23 female (age range 7-66, average 28.22) who agreed to take part in a formal evaluation of the app. The procedure was approved by the University of Abertay, Social and Health Sciences Research Ethics Committee.

\subsection{Apparatus}

The software comprises the Tapology suite of 5 mini-games, each designed to challenge a different part of the visuo-motor control of the participants.

The app makes use of the Flurry (C) Flurry) analytics API to keep track of player information in the cloud during testing. Flurry is designed to keep track of thousands of users for company analytics and marketing, and by using the event tracking system it provides, it serves the purpose of information gathering. When a player starts the app it logs them as a new player and assigns them a unique identifier for the iPad they are using. As they complete the different mini-games, they trigger events which are sent to Flurry with information regarding what just happened. For example, in the card matching game, once they make the first match, the time taken is sent to Flurry to give an idea of the user's reaction time. With the data being stored in the cloud this made analysis easy to access. 


\subsection{Procedure}

For the user trials, visitors to the official 'Tapology' launch stall at Dundee's 2013 DareProtoplay Indiefest national video games festival (Fig. 4) were invited to explore the app to assess the ease of use of the game. Those who gave informed consent were then given a short questionnaire on their game use, including number of platforms used and length of time spent on game play per month.

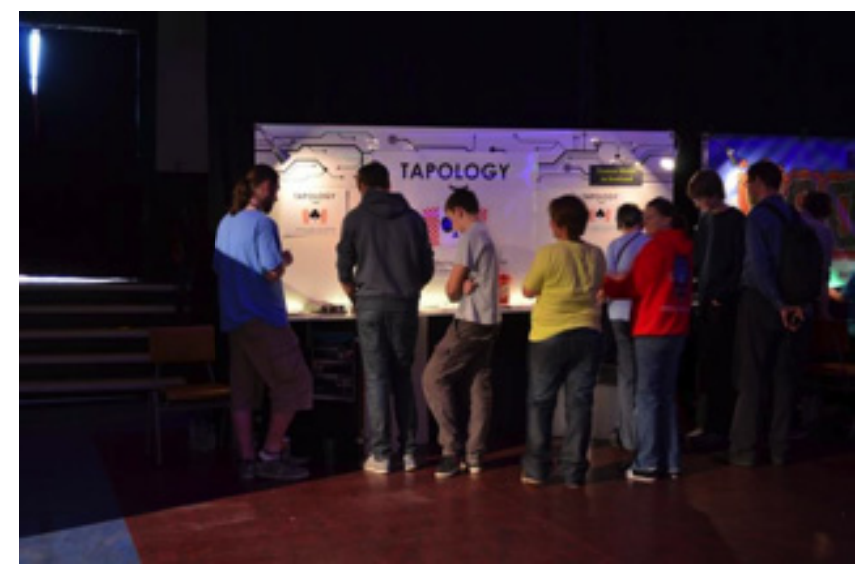

Fig. 4. Users recruited at the 'Tapology' stall at Dare Protoplay Indiefest, video games festival in Dundee

\section{$4 \quad$ Results}

Average App completion time was found to be 4.88 minutes with a mean of 3 and a max of 8 minutes (SD 1.09). Age of tester accounted for $25 \%$ of the variance in the app completion time. These durations are appropriate for a repeated regular casual game app. The key is to create a short burst of engagement that can be used on a daily basis.

Inspection of the open prompt questionnaire responses also revealed the positive reaction from users of all age groups, with over 50 individual positive statements and 25 suggestions for additional apps or improved gameplay. In addition to approving of the game play, on debrief, the users were particularly receptive to the idea of an einclusion based assistive app. An insight from this communication with users was that the app was of potential use, not just to elderly users, but also to other users with educational special needs.

Further informal acceptance testing was done with the original co-design client group and other clients from Fife Society for the Blind (Fig 5). This enabled the team to close the loop with the co-design group with the intention of fostering a sense of ownership of the project with the client group. 


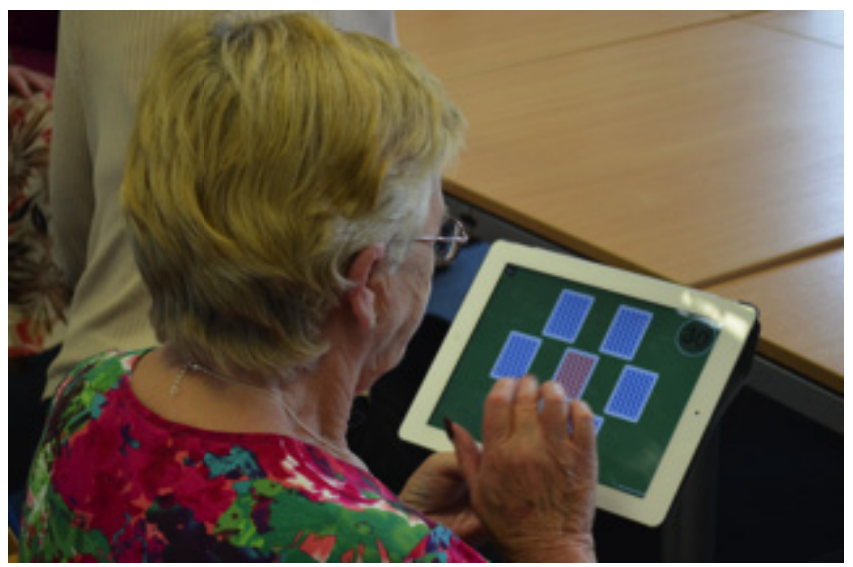

Fig. 5. Fife Society for the Blind User testing the card match app

\section{Summary}

We have co-designed a group of 5 mini-games, all designed to challenge different parts of visual cognitive function. The 'Tapology' app is an attempt to bridge the gap that can occur between diagnosis and point of impact of sight loss [1] in a constructive engaging manner. This was achieved thanks to a coordinated sequence of design events including a co-design session; conference calls; user test sessions at Dare Protoplay and at 'Road Map' meetings. Further user trials have been conducted with clients from the Fife Society for the Blind and with members of the public and the DareProtoplay video games festival. The information gained from this feedback reinforce the original concept of an assistive suite of apps with the capacity to monitor and in the longer term, rehabilitate visual function in a group of users with impaired vision and no prior experience of ICT. However, the need for social 'scaffolding' of the interactions is acknowledged, since the long term ownership and maintenance of ICT devices remains not fully accessible to low vision users.

Using pervasive, low-cost computing devices such as tablet computers for patient driven visual rehabilitation and monitoring offers health care professionals a chance to completely reorient the ownership of self-assessment of health using an engaging platform. By making the assessment protocol game-based, and delivering it in a standard household device that has additional functions and purposes beyond clinical ones, the scope of Tapology is focused on a broad e-inclusion goal. In the long term, this is to personalise healthcare assessment and to contextualise it within a wider accessibility function to enable users to gradually access communication, internet and accessibility functions associated with tablet based systems. Digital exclusion is a problem across both rural and urban Scotland [2], but low cost, easy to use tablet computers offer a pervasive solution suitable for ice breaking sessions in ways that standalone PC computers cannot deliver. In particular, the multi-touch capacity of modern tablets has brought the written word to low vision users by enabling intuitive, pinch-based scaling of text with capacitive multi-touch gestures. 


\section{Future Work}

The ultimate objective for the team is to create fully working and testable visual rehabilitation software application embedded in a more general vehicle to facilitate einclusion in low vision users. This will be achieved through a two-pronged approach. The first goal is develop the Tapology 'app' as an enabling tool for care-based and voluntary sector led e-inclusion outreach work. With a series of collaborative workshops hosted by Fife Society for the Blind and involving their clients, we aim to assess the extent to which group based activity, supported by specialists, can demonstrate how the introduction of ICT in the form of tablet computers can raise awareness about a wide range of assistive functions. Facilitation of introduction to ICT prior to adoption is critical, and the role of a trusted source in the introduction of new processes. These 'meet the ipad' sessions, facilitated by support workers and specialists will help to manage expectations about the scope of the technology and the requirements for adoption. The workshops can promote awareness of the built-in accessible features in devices such as the iPad. The ability to use Siri (@) Apple Corporation) to talk to the device and command actions, coupled with its ability to read back to the user is not widely publicized in minority end user groups, yet is of high value.

The second element of the project involves refining the gameplay and demonstrating peripheral vision improvement using the perceptual learning tasks previously shown to have potential benefit in AMD (e.g. [3]). As a complex intervention, this application will benefit from the proof of feasibility of Tapology as a vehicle for behavioural engagement in game-based studies of visual function [e.g., 12].

Acknowledgement. This project was part funded by a grant to K. Scott-Brown from Scottish Crucible, and further supported by the iAge Project (iAge:e-inclusion in ageing Europe; www.iageproject.eu). We are grateful to Alan Suttie and his colleagues and clients at the Fife Society for the Blind for facilitating co-design and evaluation sessions. We are also grateful to Caroline MacEwen from NHS Tayside for advice on the direction of focus for the application development. We are also grateful to Bela Havasreti, Mark Adams, Pauline Mack, Lesley Parker-Hamilton for facilitating the User Trial.

\section{References}

1. Thurston, M.: An enquiry into the emotional impact of sight loss and the counselling experiences and needs of blind and partially sighted adults. Counselling and Psychotherapy Research 10(1), 3-12 (2010)

2. White, D.: Across the Divide. Tackling digital exclusion. Carnegie UK Trust Report (2013),

http: / / www. carnegieuktrust.org.uk/publications/2013/

across-the-divide-full-report 
3. Yu, D., Cheung, S.-H., Gordon, E., Legge, G.E., Chung, S.: Reading speed in the peripheral visual field of older adults: Does it benefit from perceptual learning? Vision Research 50(9), 860-869 (2010)

4. Green, C.S., Bavelier, D.: Action video game experience alters the spatial resolution of attention. Psychological Science 18, 88-94 (2007)

5. Dye, M.W.G., Green, C.S., Bavelier, D.: Increasing speed of processing with action video games. Current Directions in Psychological Science 18(6), 321-326 (2009)

6. Knox, P.J., Simmers, A.J., Gray, L.S.: An exploratory study: Prolonged periods of binocular stimulation can provide an effective treatment in childhood amblyopia. Investigative Ophthalmology and Visual Science 3, 817-824 (2012)

7. Chung, S.T., Mansfield, J.S., Legge, G.E.: Psychophysics of reading. XVIII. The effect of print size on reading speed in normal peripheral vision. Vision Res. 38, 2949-2962 (1998)

8. Martinez, S., Carrillo, A.L., Scott-Brown, K., Falgueras, J.: AGILE Interface for 'NoLearning nor Experience required' Interaction. In: Martín, E., Haya, P.A., Carro, R.M. (eds.) User Modeling and Adaptation for Daily Routines. Human-Computer Interaction Series, pp. 119-151. Springer (2013)

9. Keates, S.: Pragmatic research issues confronting HCI practitioners when designing for universal access. Int. J. on Universal Access in the Information Society 5(3), 269-278 (2006)

10. Albinsson, L., Lind, M., Forsgren, O.: Co-Design: An Approach to Border Crossing, Network Innovation (2007)

11. Isaacs, J., Martinez, S., Scott-Brown, K., Milne, A., Evans, A., Gilmour, D.: Mobile Technology and E-Inclusion. In: Stephanidis, C., Antona, M. (eds.) UAHCI 2013, Part III. LNCS, vol. 8011, pp. 626-635. Springer, Heidelberg (2013)

12. Li, R., Polat, U., Makous, W., Bavelier, D.: Enhancing the contrast sensitivity function through action video game playing. Nature Neuroscience 12, 549-551 (2009) 